\title{
Two-dimensional grain boundary percolation in alloy 304 stainless steel
}

Brent L. Adams

b_I_adams@byu.edu

John A. Basinger

David T. Fullwood

dfullwood@byu.edu

E. R. Homer

Follow this and additional works at: https://scholarsarchive.byu.edu/facpub

Part of the Mechanical Engineering Commons

Original Publication Citation

Acta Materialia 54 (26) 1381-1388

\section{BYU ScholarsArchive Citation}

Adams, Brent L.; Basinger, John A.; Fullwood, David T.; and Homer, E. R., "Two-dimensional grain boundary percolation in alloy 304 stainless steel" (2005). Faculty Publications. 371.

https://scholarsarchive.byu.edu/facpub/371

This Peer-Reviewed Article is brought to you for free and open access by BYU ScholarsArchive. It has been accepted for inclusion in Faculty Publications by an authorized administrator of BYU ScholarsArchive. For more information, please contact ellen_amatangelo@byu.edu. 


\title{
Two-dimensional grain boundary percolation in alloy 304 stainless steel
}

\author{
J.A. Basinger *, E.R. Homer, D.T. Fullwood, B.L. Adams \\ Department of Mechanical Engineering, Brigham Young University, Provo, UT 84604, USA \\ Received 7 December 2004; received in revised form 4 May 2005; accepted 17 June 2005 \\ Available online 15 July 2005 \\ 2005 Acta Materialia Inc. Published by Elsevier Ltd. All rights reserved. \\ Keywords: Grain boundaries; Misorientation; Microstructure; Percolation; OIM \\ 2005 Acta Materialia Inc. Published by Elsevier Ltd. All rights reserved. \\ doi:10.1016/j.scriptamat.2005.06.026 \\ * Corresponding author. Tel.: +1 801422 2059/3753219; fax: +1 8014220516. \\ E-mail address:drjayb20@yahoo.com (J.A. Basinger). \\ www.actamat-journals.com \\ Scripta Materialia 53 (2005) 959-963
}

\begin{abstract}
An experimentally-obtained percolation threshold for high-angle random grain boundary networks in alloy 304 stainless steel is compared to thresholds predicted by percolation theory. A discrepancy occurs in the two values ( 0.46 experimental and 0.65 theoretical). Possible reasons for the discrepancy are explored. The grain boundary network appears to be composed of two distinct sub-networks, with the 'outer' network acting as the dominant contributor to the percolating paths.
\end{abstract}

\section{Introduction}

Percolation models are an attractive approach to modeling certain defect-sensitive properties of polycrystalline materials. The connectivity of grain boundary networks has been described in the context of idealized lattice-based models in order to obtain a percolation threshold at which a connected set of defective boundaries spans the model. This percolation threshold may then be associated with certain critical phenomena, including intergranular failure. Recent work in modeling percolation of grain boundaries introduces the local crystallographic constraints, thus bringing the lattice based models closer to real polycrystals [1-3].

However, little experimental data has been generated for comparison with the idealized case. This paper analyses such a data set for 304 stainless steel.

The 2-D data set was obtained by orientation imaging microscopy (OIM) using a conventional approach [4]. The resultant network of grain boundaries was analyzed, and each section classified as either high-angle random (HAR) or non-HAR. High-angle random boundaries are assumed to be 'defective' (e.g. susceptible to intergranular failure) and labeled as 'open'. All other boundary (bond) types, including low-angle grain boundaries and coincidence site lattice boundaries (CSLs) with $\Sigma \leq 29$ are presumed immune to 'failure' and labeled 'closed'. The method is consistent with previous work [5-7]. 


\section{Experimental approach}

OIM data was gathered from a single section plane of alloy 304 stainless steel at a step size of $6 \mathrm{~lm}$ between hexagonally spaced data points. This material is interesting because it can be produced with a high fraction of twin/CSL boundaries that are believed to break up grain boundary networks of susceptible boundaries $[5,6]$. The data set covers an area of $4 \cdot 4 \mathrm{~mm}$, with an average grain size of $89 \mu \mathrm{m}$. A total of 1361 grains appear in the scan. Scan data were then 'virtually annealed', using a standard method in OIM ${ }^{\mathrm{TM}}$, to reduce the occurrence of grain boundary artifacts and to thin them to sizes less than the step size. Each data point taken contains $x, y$ coordinates as well as the three Euler angles, $\varphi_{1}, \varphi, \varphi_{2}$ depicting the local orientation of the crystal lattice. Each pair of OIM points gives rise to a data point that either contributes to the graph (i.e. it represents a segment of a grain boundary) or lies within a grain and does not contribute. The character of the data point is determined from indexing the electron backscattering diffraction patterns at each pair of points; if the difference in crystallographic angle between the two points is less than $5^{\circ}$, the points are assumed to lie within a common grain. A bigger difference in the respective angles indicates the presence of a grain boundary between the points.

It is acknowledged that a percentage of LAR boundaries with angles less than $5^{\circ}$ will be excluded from the analysis and add some bias to the results.

From this construction a list of points associated with HAR grain boundaries (open bonds) and non-HAR grain boundaries (closed bonds) is generated. The fraction of open points to total grain boundary points is effectively a length-based assessment of the ratio of open bonds to total bonds, and was readily generated by the computer. A visual assessment of the data was subsequently performed in which a bond was defined as a section of grain boundary between two vertices, or intersections. This second approach is a topological, rather than length based, assessment, and is the appropriate measure for comparison with published percolation data.

The crystallographic criteria used to delineate the two classes of boundaries can be altered with the consequence that the ratio of numbers of susceptible bonds to the total is varied in the model. By varying the $N$-parameter for allowable angular deviation in CSLs, $\Delta \theta \leq 15 \Sigma^{-N}$, over the range $1 / 2 \leq N \leq 1$, the fraction of susceptible boundaries was adjusted. The greater the value of $N$, the more restrictive the criterion becomes, and the greater the number of open bonds. Note that $N=1 / 2$ corresponds to Brandon's criterion [7] and $N=5 / 6$ to the Palumbo-Aust criterion [8].

The sizes of (connected) clusters of open bonds are determined, and an assessment is made as to whether percolation has occurred across the entire dataset. The fraction, $p$, of open bonds to the total number of bonds in the graph is also noted. $N$ is increased, and when the number fraction of susceptible bonds, $p$, reaches the lowest level at which percolation across the entire lattice occurs (in both directions), the percolation threshold is reached for the (finite) model; $p=p_{\mathrm{c}}$, and $p_{\mathrm{c}}$ is an approximation to the true percolation threshold in the infinite lattice (note that the error in this approximation may be significantly affected by the finite sample size, as is discussed later). 


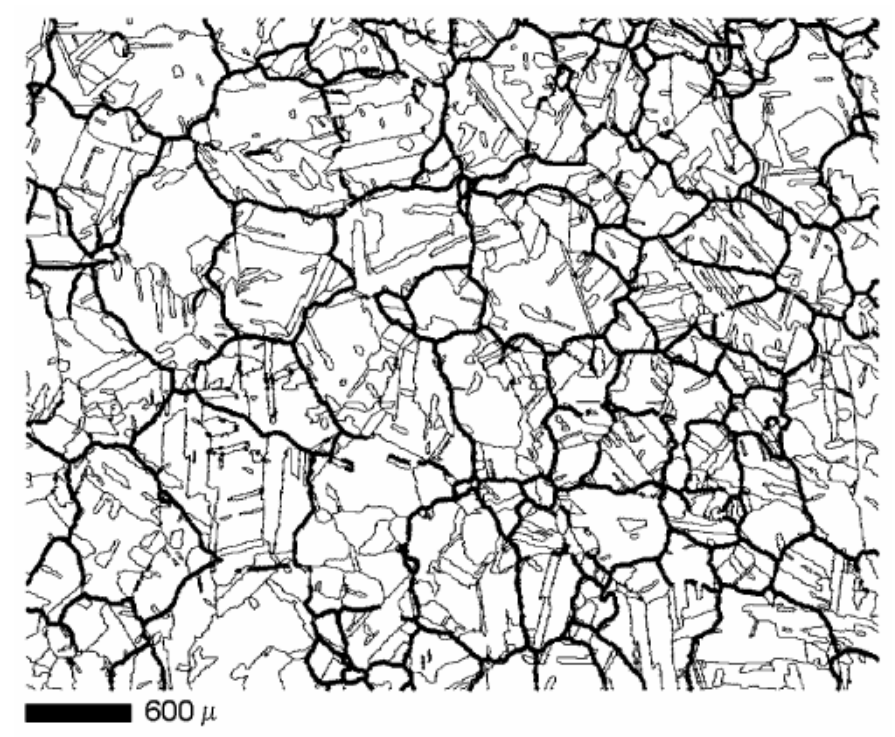

Fig. 1. Reconstructed grain boundary map from an OIM scan of alloy 304 stainless steel. The thick lines indicate susceptible boundaries with a rotation angle greater than $15^{\circ}$. The thin lines indicate low angle and CSL boundaries. The 'resistant' boundaries can be seen to break up connectivity of the 'susceptible' grain boundaries.

Fig. 1 shows an OIM scan with the non-HAR (closed) boundaries as thin lines and the HAR (open) boundaries above $15^{\circ}$ of misorientation as thick lines. Fig. $2 \mathrm{a}-\mathrm{c}$ display the largest cluster of only open bonds for a given value of $N$. A cluster that spans the entire window is a percolating cluster.

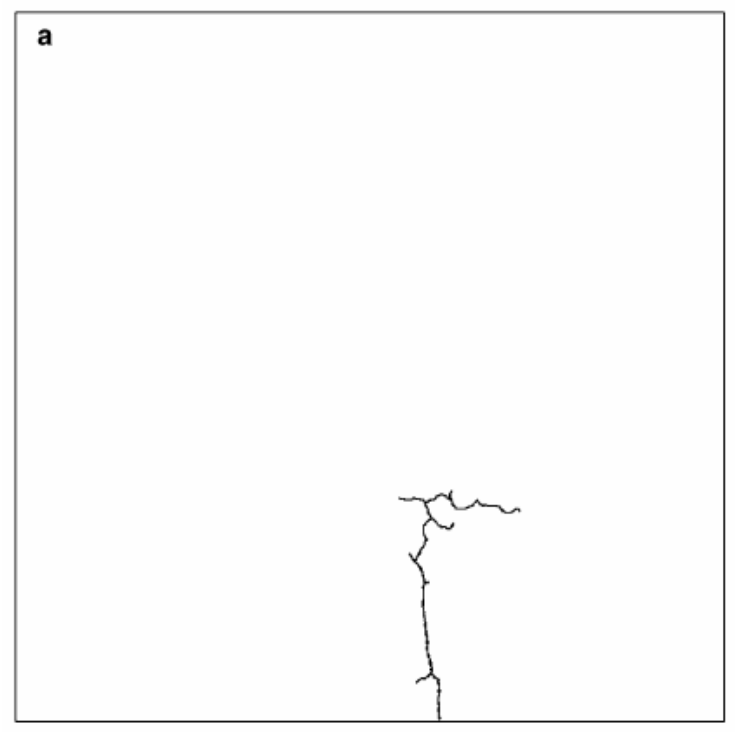

$600 \mu$

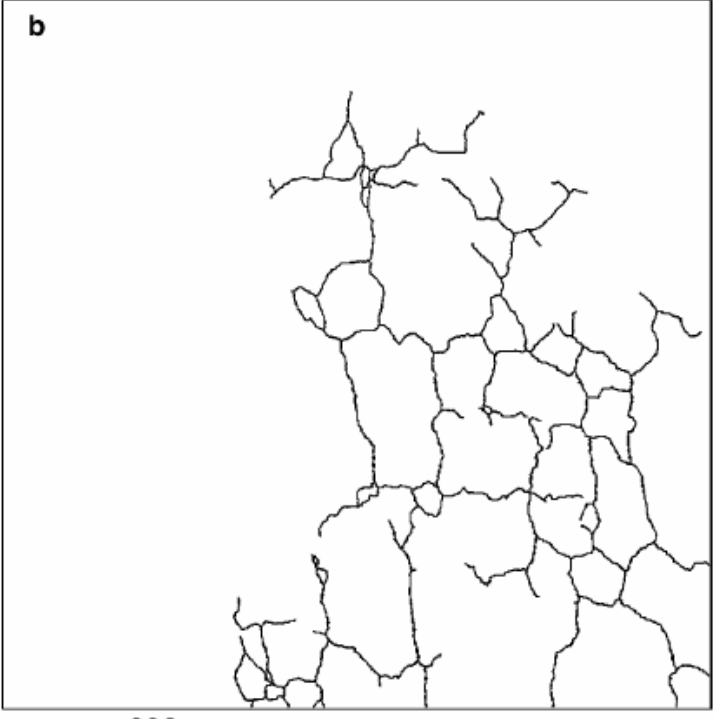

$600 \mu$ 


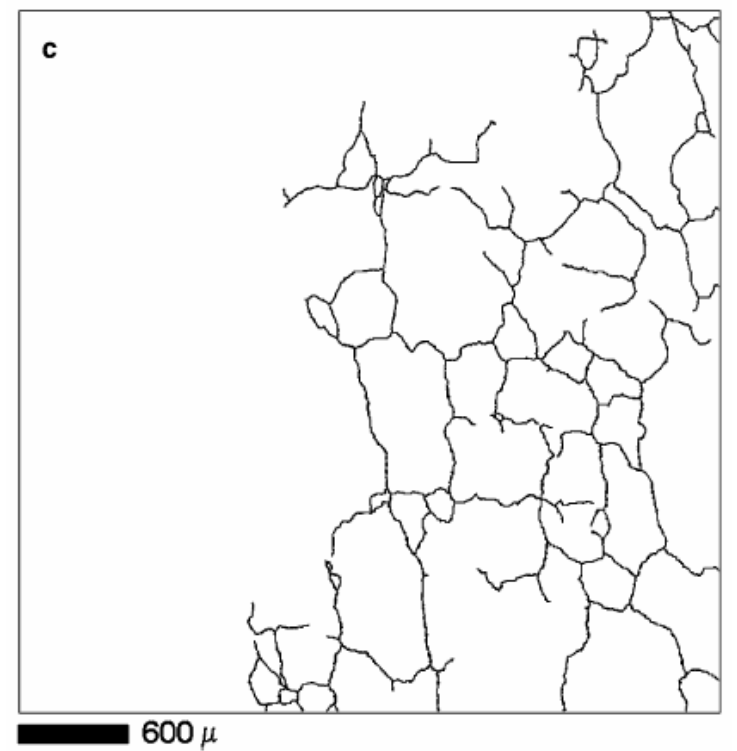

The main results of the present work are shown in Table 1. Percolation is observed when the $N$-parameter reaches a value of 0.9013 . At this value of $N$ the fraction of $\Sigma 3$ boundaries out of the total CSL boundaries is 0.87 , and none of the non-susceptible bonds are LAR; the fraction of open bonds is 0.46 , i.e. $p_{\mathrm{c}}=0.46$.

$\begin{aligned} & \text { Table 1 } \\
& p \text { (HAR) vs } N \text { for 'length-based' and 'topological' measures on the grain } \\
& \text { boundary structure (values at the percolation threshold in bold) }\end{aligned}$
\begin{tabular}{llllll}
$N$ & & & & \\
\cline { 2 - 6 } & .5 & .8333 & .9 & .9013 & .95 \\
\hline $\begin{array}{c}\text { p(open) } \\
\text { (length-based) } \\
\text { p(open) } \\
\text { (topological) }\end{array}$ & 0.1861 & 0.2283 & 0.2342 & $\mathbf{0 . 2 3 4 3}$ & 0.2390 \\
& & & & $\mathbf{0 . 4 6}$ & \\
& & & & $\begin{array}{l}\text { Percolation } \\
\text { threshold }\end{array}$ \\
\hline
\end{tabular}

It should be noted that during the calculation of the fraction of open bonds, loops on the grain boundary network that do not contribute to paths between vertices are ignored (these account for almost $20 \%$ of the total bonds).

When the ratio of 'total length of open grain boundary' to 'total grain boundary length' is measured (as counted by the computer from the original OIM data) the percolation threshold is found to be 0.2343 . This value is not very meaningful when compared with standard percolation theory where the number of bonds is the important parameter, and length is irrelevant. However, the measure gives an indication of the difference in length between the average open and closed bond.

As a further exercise, the original hexagonal lattice used for the OIM scan was taken as the basis for the percolation results. The grain boundary network is effectively mapped onto a standard hexagonal lattice. Any lattice bond that does not coincide with a HAR segment is labeled 'closed' thus introducing a large number of extra closed bonds ('zero misorientation' grain boundaries. 
The resultant percolation threshold is 0.01907 . This demonstrates why a real grain boundary network cannot be mapped directly onto a standard lattice; the number of extra paths introduced by drawing the lattice over the network heavily biases the result. The network must be mapped in a topological manner to preserve the essential path characteristic.

\section{Discussion}

Model percolation thresholds for bond-type percolation on hexagonal lattices have been defined for standard as well as constrained cases. Stauffer and Aharony cite a percolation threshold of $p_{\mathrm{c}}=0.653$ for the 2-D hexagonal lattice [9]. In the constrained models [10] Schuh and Frary put the percolation threshold at $\sim 0.659$ (using the result for 'twinned' material).

The observed value of $p_{\mathrm{c}}=0.46$ is significantly lower than these standard lattice results.

Even if we abandon lattice-based models for random structures it is expected that a 2-D network will percolate at approximately two open bonds at each vertex. For 3-D the value is 1.5 bonds (see [11] for example). The 2-D value translates to a percolation threshold of 0.67 for a structure made up of triple junctions (as is the case for grain boundaries). Again, this is significantly above the result of 0.46 . Several factors may contribute to these discrepancies:

1. The finite size of the sample will lead to a lower percolation threshold than that of the theoretical infinite lattice. Calculations on a hexagonal lattice of approximately the same number of grains as the sample (approximately 400 with the non-contributing loops excluded) give a drop in $p_{\mathrm{c}}$ from 0.653 (infinite lattice) to 0.608 (finite lattice). Furthermore, the lowest probability at which percolation occurred (in a single direction) over several thousand trials on this finite lattice was 0.502 . The mean 'lowest percolation' probability over 20 scans of the lattice was 0.541 with standard deviation of 0.02 ; the value of 0.46 is more than 3 standard deviations from this mean. Hence, while the finite sample size will certainly contribute significantly to the shift in percolation threshold, it is extremely unlikely that the sample is simply at the edge of the statistical distribution of data that follows the random hexagonal lattice.

2. The standard hexagonal lattice may not be a good representation (from a network point of view) of a 2-D grain boundary structure. While the hexagonal lattice is composed of triple junctions at each vertex (which is similar to a grain structure), the random number of edges to each grain is likely to have a significant effect on the percolation threshold. However, we have argued that even for random networks the percolation threshold should be near 0.67 . This is discussed further in a separate paper [12] where it is found that the 2-D grain structure should in fact have a higher threshold than predicted by the hexagonal lattice (not a lower one, as found here). 
3. The high-angle grain boundaries (open bonds) may not be randomly placed throughout the structure.

4. Related to the previous point, a large number of subgrains or twins may lead to a bias towards low-angle/ CSL boundaries (or closed bonds) which are not randomly distributed, and hence do not contribute to percolation to the same extent as the high-angle boundaries. These subgrains bonds form clusters of closed bonds that are not crossed by the HAR open bonds, and effectively simply add weight to the fraction of closed bonds.

The threshold value of 0.23 , based upon total length of bond segments, highlights that there appears to be two very different length scales in the grain boundary population. The value of 0.23 includes LAR/CSL grain boundary loops that were factored out of the 0.46 result (as described above), and also add to the bias. A rough adjustment to remove the loops puts the length-based threshold at 0.30 - still well below the value of 0.46 . This indicates that the average length of the HAR bonds is 0.35 times the average length of the non-HAR bonds. There appears to be a network of non-HAR boundaries that falls largely in the interior of the HAR network, and regularly breaking up the HAR graph into shorter length bonds. We will label the inner network as 'noncontributing', and will assume that the inner network breaks up HAR and non-HAR boundaries of the 'contributing' network to the same degree. If we now assume that the percolation threshold for the contributing network is 0.67 , then the length of the contributing network is $34 \%$ of the total grain boundary length in the sample.

From careful consideration of the 2-D grain structure, and taking into account the discussion about length scales above, point 4 appears to be the dominant reason for the low percolation value. A criterion for sifting out subgrains would be required to arrive at a better fit to standard percolation theory. It should also be noted that the process of increasing the $N$ value to increase the fraction of high-angle boundaries (or open bonds) is an artificial way of increasing the percentage. It does not add open bonds in a random manner; and in fact, it can be seen from the figures that the outcome was generally the completing of 'partially' open bonds. Since this is the case, it would seem that the best value for $N$ is one that provides a definite separation between open and closed bondsi.e. reduces the number of bonds that are made up of both high and low angle segments. The value of 0.9013 used at the percolation threshold appears to be a reasonable value from this point of view.

\section{Concluding remarks}

Percolation of HAR boundaries in the sample steel has been found to occur at 0.46 . This is significantly lower than theory predicts (using either the rough calculation of 0.67 , or lattice-based results). Various observations persuade us that this is not simply the result of a statistical anomaly (although the finite sample size will contribute significantly to the shift), but is likely to be mainly due to a significant number of LAR/CSL subgrain boundaries that do not constitute a truly random population. These are characterized by clusters of non-HAR boundaries that cannot contribute to the percolation process due to the lack of open bonds (see Fig. 1). 
The HAR boundaries themselves may also not be randomly distributed, but the subgrains would have to be filtered out of the picture first to determine this. Thus, there is currently no evidence to suggest that the HAR boundaries are not randomly distributed. It is interesting to note, however, that the application of the triple junction constraint to the standard lattice model [3] reduces the random nature of the HAR distribution and affects the percolation threshold; according to [3], the triple junction constraint tends to cluster the HAR boundaries in a non-random manner. There may be other constraints - for example, based upon processing conditions - that affect the random nature of the network.

Consideration of the total length of HAR and non- HAR boundaries at percolation (which is a measure readily obtained from OIM scans) gives a percolation threshold of 0.23 , demonstrating that the average length of HAR bonds is less than that of non-HAR bonds. Hence caution should be exercised if length-based measures are used. For comparison with percolation theory the average length of HAR and non-HAR bonds (i.e. the distance between triple junctions) must be the same. It may be that the difference in the average length of the two types of bond gives some kind of measure on the percentage of subgrains, and can be used to scale the percolation threshold to a value more in line with standard theory (i.e. the scaling factor would reduce the contribution from the subgrain boundaries that contribute less to the percolation process).

\section{Acknowledgement}

This work was supported by the MRSEC program of the National Science Foundation under Award Number DMR-0079996.

\section{References}

[1] Minich RW, Schuh CA, Kumar M. Phys Rev B 2002;66:052101.

[2] Frary M, Schuh CA. Appl Phys Lett 2003;83:3755.

[3] Frary M, Schuh CA. Phys Rev B 2004;69:134115.

[4] Adams BL, Wright SI, Kunze K. Metall Trans 1993;24A:819.

[5] Watanabe T, Murakami T, Karashima S. Scripta Metall 1978;12: 361.

[6] Palumbo G, King PJ, Aust KT, Erb U, Lichtenberger PC. Scripta Metall 1991;25:1775.

[7] Lin P, Palumbo G, Erb U, Aust KT. Scripta Metall Mater 1995;33:1387.

[8] Brandon DG. Acta Metall 1966;14(Nov):1479.

[9] Stauffer D, Aharony A. Introduction to Percolation Theory. $2^{\text {nd }}$ ed. Bristol, PA: Taylor and Francis; 1992. p. 17. 
[10] Frary M, Schuh CA. Connectivity and percolation behaviour of grain boundary networks in three dimensions, Philos Mag, in press.

[11] Janzen J. J Appl Phys 1975;46:966.

[12] Fullwood DT, Basinger JA, Adams BL, Lattice-based structures for studying percolation in 2-D grain networks, submitted for publication. J.A. Basinger et al. / Scripta Materialia 53 (2005) 959-963963 